\title{
MR diagnosis of cranial neuritis focusing on facial neuritis: Performance of contrast-enhanced 3D-FLAIR technique
}

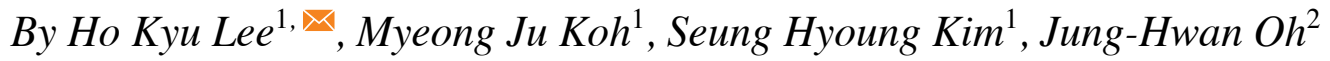 \\ Departments of ${ }^{1}$ Radiology and ${ }^{2}$ Neurology, Jeju National University, Jeju City, Republic of Korea
}

\begin{abstract}
Our purpose was to evaluate usefulness of the contrast-enhanced 3 dimensional fluid attenuated inversion recovery (3D-FLAIR) technique of half brain volume to diagnose the patients with facial neuritis based on segment-based analysis. We assessed retrospectively 17 consecutive patients who underwent brain MR imaging at 3 tesla for facial neuritis: 11 patients with idiopathic facial neuritis and 6 with herpes zoster oticus. Contrast enhanced 3D-FLAIR sequences of the half brain volume were analyzed and 3D T1-weighted sequence of the full brain volume were used as the base-line exam. Enhancement of the facial nerve was determined in each segment of 5 facial nerve segments by two radiologists. Sensitivity, specificity and accuracy of enhancement of each segment were assessed. The authors experienced a prompt fuzzy CSF enhancement in the fundus of the internal auditory canal in patients with enhancement of the canalicular segment. Interobserver agreement of CE 3D-FLAIR was excellent ( $k$-value 0.885). Sensitivity, specificity, and accuracy of each segment are 1.0, 0.823, 0.912 in the canalicular segment; $0.118,1.0,0.559$ in the labyrinthine segment; $0.823,0.294,0.559$ in the anterior genu; $0.823,0.529,0.676$ in the tympanic segment; $0.823,0.235,0.529$ in the mastoid segment, respectively. In addition, those of prompt fuzzy enhancement were 0.647 , 1.0 , and 0.824 , respectively. Incidence of prompt fuzzy enhancement with enhancement of the canalicular segment was 11 sites (55\%): 6 (54.5\%) in idiopathic facial neuritis and $5(83.3 \%)$ in herpes zoster. Enhancement of the canalicular segment and prompt fuzzy enhancement on CE 3D-FLAIR was significantly correlated with occurrence of facial neuritis $(p<0.001)$. CE 3D-FLAIR technique of the half brain volume is useful to evaluate the patients with facial neuritis as an adjunct sequence in addition to contrast-enhanced 3D T1-weighted sequence. On segment-based analysis, contrast enhancement of the canalicular segment is the most reliable. Prompt fuzzy enhancement is seen in not only herpes zoster, but in idiopathic facial neuritis.
\end{abstract}

Key words: Bell's palsy, Herpes zoster oticus, Cranial neuritis, MRI, Facial nerve

\section{INTRODUCTION}

Cranial neuritis can occur as mono- or polyneuropathy. State-of the art magnetic resonance (MR) imaging technique have made it possible to reproduce the cranial ner-

Received: February 22, 2019; Revised: April 1, 2019; Accepted: April 3, 2019 $\checkmark$ Correspondence to : Ho Kyu Lee

Department of Radiology, Jeju National University Hospital, 15 Aran 13-gil, Jeju-City 63241, Republic of Korea

Tel: 82-64-717-1373, FAX: 82-64-7171-1370

E-mail: hoklee33@gmail.com ves for accurate diagnoses. 3D MR technique provides a greater spatial resolution with or without the use of gadolinium contrast agent, allowing radiologists to diagnose disease conditions that involves the small cranial nerves such as the oculomotor, abducens, facial, and vestibulocochlear nerves. ${ }^{1-4)}$

The ability of the contrast enhanced 3D fluid attenuated inversion recovery (CE 3D-FLAIR) sequence to find minute signal changes mainly results from the high sensitivity of FLAIR imaging to subtle changes of longitudinal magnetization of the contrast agent in the fluid space, 
which declines with higher concentration of the contrast agent. $^{5,6)}$ It has been reported that FLAIR imaging can detect a concentration of gadolinium four times lower than that detectable by contrast-enhanced 3 dimensional T1weighted sequence (CE 3D-T1WS). ${ }^{7)}$

Facial neuritis is mostly caused by herpes simplex virus which induces Bell's palsy, and secondarily by varicella zoster virus which induces Ramsay-Hunt syndrome. ${ }^{2)}$

MR imaging of patients with facial neuritis is usually indicated for patients with facial palsy who have atypical manifestations and those with intractable palsy despite therapy. To diagnosis and predict outcomes in patients with facial neuritis, CE 3D-FLAIR sequence has been a potent MR sequence than CE 3D-T1WS since it has higher specificity and accuracy., ${ }^{3,4)}$

The purpose was to evaluate usefulness of the CE 3DFLAIR technique of the half brain volume to diagnose the patients with facial neuritis based on segment-based analysis.

\section{MATERIALS AND METHODS}

This retrospective study was approved by our institutional review board for human investigation and informed consent was waived. We initially selected consecutive patients who presented with facial palsy at our institution between January 2015 and October 2017 and underwent brain MRI examination. Among these patients were excluded the following conditions: infection of the central nervous system (CNS), underlying malignancy, recent trauma at symptomatic side, or a previous history of temporal bone surgery. Thus all 17 patients consists of 11 idiopathic facial neuritis (male $:$ female $=6: 5$, mean age 49.6 years, range $19 \sim 67$ years) and 6 herpes zoster oticus (male : female $=2: 4$, mean age 57.7 years, range $37 \sim 76$ years). Mean time interval between symptom onset and MRI examination was 13 days (range 2 30 days). All 17 patients had been diagnosed with unilateral facial neuritis by neuronal examination. MR imaging was performed to exclude a neoplastic or CNS cause of facial palsy, other combined pathology. Initial House-Brackman (HB) scale which is widely used grading system of facial nerve $(\mathrm{CN}$ VII) paralysis was IV in 7, III in 7, II in 2 and V in one patient (average: 3.4$){ }^{8)}$

\section{MR Examination}

All MR images were performed with a 3 T MR unit
(Intera Achieva: Philip Medical Systems, Best, the Netherlands) using an phased array SENSE head coil. MR images included for image analysis were 3D-FLAIR sequences using gadolinium-diethyle-triaminepentaacetic acid (Gd-DTPA: Gadovist, Bayer, Germany) at $0.1 \mathrm{mmol} / \mathrm{kg}$ of body weight in $8 \sim 10$ minute delay after the injection of the contrast. The imaging slab was the lower half of the brain from the craniovertebral junction to the thalamus which can show all cranial nerves including facial nerves.

The parameters for CE 3D-FLAIR of the half volume of the brain were as follows: Phillips MRI scanner- VISTAFLAIR sequences: TR, $4800 \mathrm{~ms}$; TE, 296 526 ms; TI, $1650 \mathrm{~ms}$; modulation of flip angle, $90^{\circ}$; section thickness, 1.2 1.5 mm; overcontiguous sections; field of view (FOV), $250 \times 250 \mathrm{~mm}$; matrix size, $320 \times 227$; number of excitation (NEX), 1; sensitivity encoding (SENSE) factor, 2; and acquisition time, 3 minutes $50 \mathrm{sec}$.

CE 3D-T1WS of the whole brain was performed in 3 minute after the injection of the contrast: Phillips MRI scanner-TR, $8 \mathrm{~ms}$; TE $4 \mathrm{~ms}$; modulation of flip angle, $13^{\circ}$; section thickness, $1.5 \sim 2.0 \mathrm{~mm}$; overcontiguous sections; FOV, $230 \times 230 \mathrm{~mm}$; matrix size, $256 \times 256$; NEX, 1; SENSE factor, 2; and acquisition time, 4 minutes $2 \mathrm{sec}$.

\section{MR image analysis}

Each facial nerve was divided into 5 segments: the canalicular, labyrinthine, anterior genu (geniculate ganglion), tympanic, and mastoid segments. ${ }^{9)}$ We used two-point rating scale: 0 , non- or mild enhancement; 1 , definite enhancement of the facial nerve in liner or fuzzy pattern (Fig. 1). CE 3D-T1WS of the whole brain volume was used as the base-line exam.

The pattern of enhancement was visually assessed by 2 neuroradiologists for 34 temporal bones with the readers unaware of the affected side and underlying cause. After a training interpretation session of total 33 patients without facial neuritis, and selected from 17 patients' data by random sampling, two readers independently reviewed pre- and post-enhanced 3D-FLAIR sequences at one week interval. The 2 datasets for each patient were randomly interpreted during different sessions. We evaluated interobserver agreement between the 2 readers using $\kappa$ statistics.

For statistic interpretation, MedCalc for Windows (version 17.2 Software bvda, Ostend, Belgium) was used to evaluate the statistics.

The sensitivity, specificity, and overall accuracy of each segment were calculated using the criteria and overall accuracy of each segment. Chi-squared test was used 

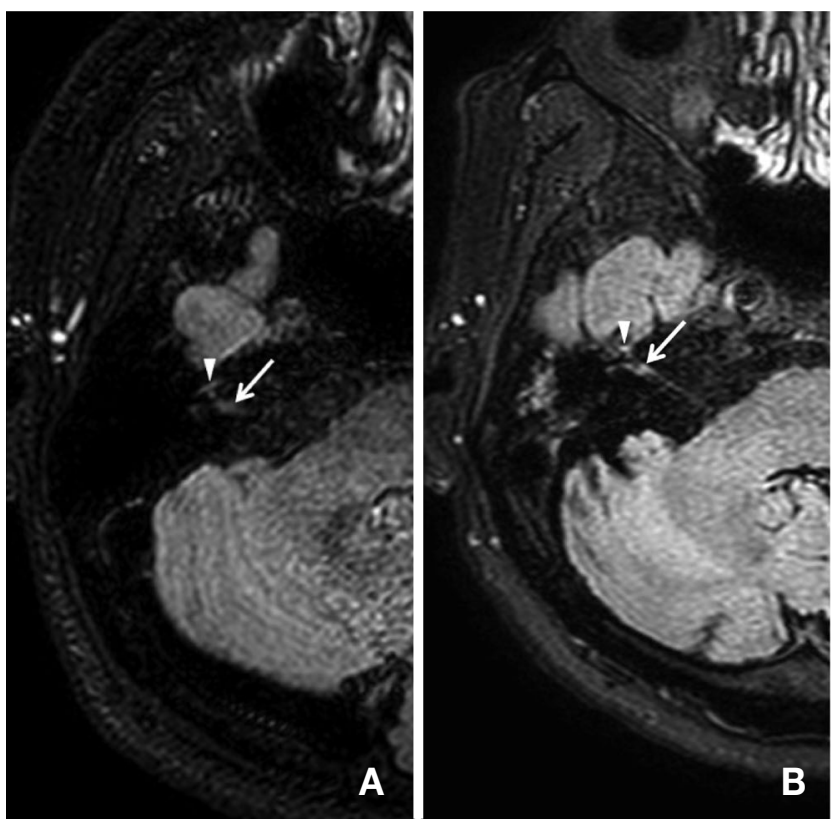

Figure 1. Pattern of enhancement of the facial nerve on contrast enhanced 3D-FLAIR images in the patient with facial neuritis. A. Non-enhancement, B. Linear enhancement of the right facial nerve at the canalicular segment (arrows = canalicular segment, arrow heads $=$ anterior genu $)$.
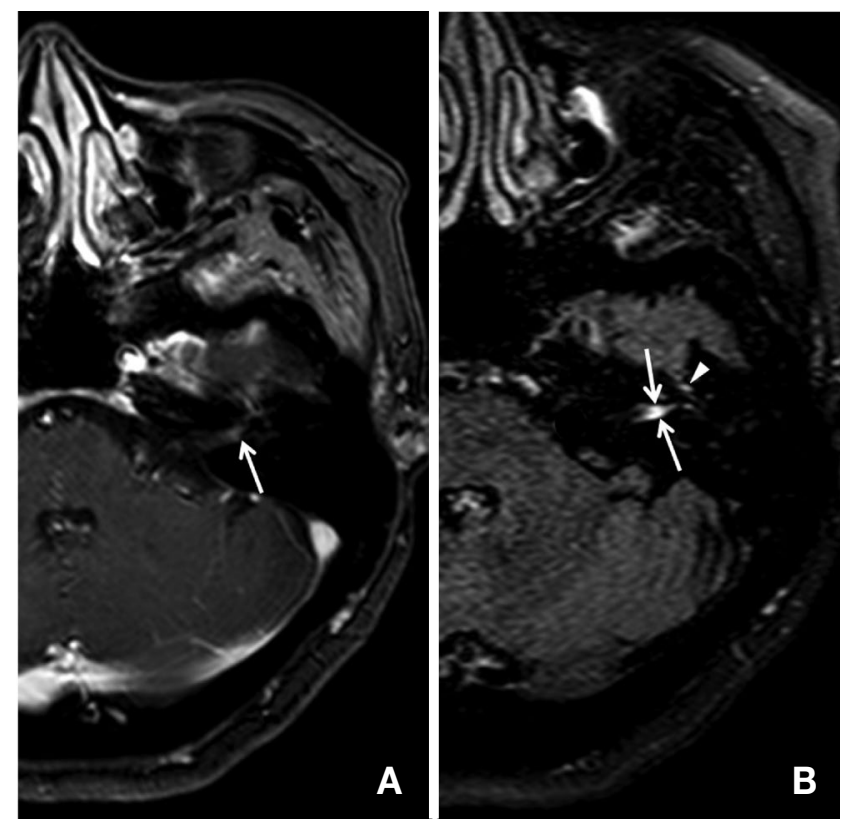

Figure 2. "Prompt fuzzy enhancement" of the facial nerve in the 55 year-old patient with left facial neuritis. A. faint dot enhancement of the facial nerve on CE T1-WS, B. In the same patient, prompt fuzzy CSF enhancement in the fundus of the internal auditory canal (arrows $=$ canalicular segment, arrow heads $=$ anterior genu).

Table 1. Results of each segment of the facial nerve on segment-based analysis

\begin{tabular}{lcccccc}
\hline & Canalicular & (Fundus*) & Labyrinthine & Anterior genu & Tympanic & Mastoid \\
\hline Sensitivity & 1.0 & 0.647 & 0.118 & 0.823 & 0.823 & 0.823 \\
Specificity & 0.823 & 0.1 & 1.0 & 0.294 & 0.529 \\
Accuracy & 0.912 & 0.824 & 0.559 & 0.559 & 0.676 & 0.235 \\
\hline p-value & $<0.001$ & 0.001 & 0.1510 & 0.4257 & 0.0339 \\
\hline
\end{tabular}

Fundus*: CSF enhancement at the fundus of the internal auditory canal called "prompt fuzzy enhancement", it belongs to the canalicular segment.

to evaluate the association between the enhancement of each segment and the facial neuritis on CE 3D-FLAIR sequence. We assessed the incidence of prompt intense CSF enhancement in the fundus of the internal auditory canal. ${ }^{10)}$ In every statistical analysis, a $p$-value $<0.001$ was considered statistically significant.

\section{RESULTS}

Interobserver agreements between two readers were excellent ( $\kappa$-value $0.885,95 \%$ CI: $0.759 \sim 1.000$ ). The sensitivity, specificity, and accuracy for detection of each segment (Table 1) on 3D-FLAIR were as followed: 1.000 , $0.823,0.912$ in the canalicular segment; $0.118,1.000$,
0.559 in the labyrinthine segment; $0.823,0.294,0.559$ in the anterior genu; $0.529,0.823,0.676$ in the tympanic segment; $0.235,0.823$, and 0.529 in the mastoid segment, respectively.

Among 20 sites showing enhancement of the canalicular segment, there was blurred or fuzzy enhancement of the facial nerve including the adjacent CSF space at the fundus of the internal auditory canal in 11 sites (55\%): $6(54.5 \%)$ in idiopathic facial neuritis and $5(83.3 \%)$ in herpes zoster. The sensitivity, specificity, and accuracy of "prompt fuzzy enhancement" were $0.647,1.000$, and 0.824 , respectively (Table 1: Fig 2).

Correlation between the enhancement of each segment of a facial nerve on CE 3D-FLAIR and occurrence of facial neuritis (Table 1) was significantly correlated with 
the presence of enhancement in both the canalicular segment $(\mathrm{p}<0.001)$ and "prompt fuzzy enhancement" $(\mathrm{p}=0.001)$.

\section{DISCUSSION}

Our results indicate that CE 3D-FLAIR sequence has an advantage in diagnosing the pathologic enhancement of the cranial nerves, and especially, facial nerve with specificity. Enhancement of normal facial nerves on conventional T1-weighted MR imaging is well known and can be explained by the flux of contrast material in the abundant circumferential arteriovenous plexus, which supplies the facial nerve in the facial canal. The vascularity of the arteriovenous plexus is most lush in the geniculate ganglion-greater superficial petrosal nerve which means the anterior genu, and in the tympanic and mastoid segments of the facial canal with the capillary component especially prominent in the geniculate-proximal greater superficial nerve segment. ${ }^{11)}$ But the vascularity abruptly terminates at the labyrinthine segment. ${ }^{12)}$ This normal enhancement of the facial nerve often hinders the clinical use of conventional MR imaging in clinical practice both diagnostic purposes and for predicting outcomes despite its high sensitivity. ${ }^{13)}$ The FLAIR sequence is more sensitive to lower gadolinium concentrations and less sensitive to higher gadolinium concentrations than T1-weighted sequence. ${ }^{14)}$ From these characteristics of FLAIR sequence, the prominent contrast enhancement of circumferential arteriovenous plexus surrounding the normal facial nerve was no longer visible on CE 3D-FLAIR sequences, which resulted in higher sensitivity and overall diagnostic accuracy compared with 3D-T1WS. ${ }^{6}$

Since this technique has the advantage over 2D-FLAIR in SNR, CNR ${ }^{15)}$ and CSF flow artifacts ${ }^{13,16)}$, 3D-FLAIR is recommended for the evaluation of patients with facial neuritis. ${ }^{3)}$ The authors chose the imaging slab of 3D-FLAIR confined to the brain stem-lower half of the brain in order to reduce the scan time, the acquisition time of it was $60 \sim 75 \%$ of the time of the same technique covering the full brain. CE 3D-FLAIR confined to the brain stem is supposed to be an useful adjunct sequence in the evaluation of either facial neuritis or other cranial neuropathy in addition to routine MR sequences covering the whole brain.

On segment-based analysis, the canalicular segment is known to be significantly correlated with the occurrence of facial neuritis, which result coincided with our study. On the contrary, in our results the enhancement of the anterior genu is not significantly correlated with the occurrence of facial neuritis, since it is probably related to different MR parameters such as thicker slice thickness and less reliability. The labyrinthine segment was not significantly correlated with the facial neuritis in both $\mathrm{Lim} \mathrm{HK}^{3)}$ and our studies. It could be due to small size of the fallopian canal and possible susceptibility artifact from the surrounding petrous bone, which results in relatively low signal-tonoise ratio and contrast-noise ratio in that area. ${ }^{3)}$

It has been reported that CSF in the fundus of the internal auditory canal in healthy subjects was enhanced 4 hours after gadolinium injection. ${ }^{16)}$ In our study, "prompt fuzzy CSF enhancement in the fundus" seen in our study after $8 \sim 10$ minutes after gadolinium injection indicates pathologic leaks caused by viral neuritis. Naganawa et al. initially reported that "prompt contrast enhancement of CSF" in the fundus of the internal auditory canal on CE 3D-FLAIR was seen in Ramsay-Hunt syndrome. ${ }^{10)}$ He proposed that Ramsay-Hunt syndrome, active inflammation in response to viral infection, might have influenced the permeability of the nerve. In our study, six patients with idiopathic facial neuritis showed "prompt fuzzy enhancement". This is first report that "prompt fuzzy enhancement in the fundus" is seen in the patients with idiopathic facial neuritis. We speculated that pathophysiology of "prompt fuzzy enhancement in the fundus" in idiopathic facial neuritis is similar to that of Ramsay-Hunt syndrome. Different incidence between two conditions could be related to activity grade of facial neuritis, scan delay time and different MR parameters than each intrinsic pathophysiology. The authors proposed that presence of "prompt fuzzy enhancement in the fundus" in the patient with facial neuritis indicates higher specificity of it.

Our study has a few limitations. First, small sample size of facial neuritis may reduce the power of the statistic analysis, Second, interval from onset of facial palsy to timing to MR imaging which range was wide from $2 \sim 30$ days may reduce reliability.

For evaluating the patient with facial neuritis, CE 3D-T1WS is not fully accepted as the standard exam due to relatively lower specificity of enhancement of the cranial nerves. ${ }^{3)}$ Therefore, CE 3D-FLAIR would help to improve specificity of MRI exam for facial neuritis. Using CE 3D-FLAIR of the half brain volume, further MRI study 
would be carried out for other cranial neuritis in addition to facial neuritis.

In conclusion, CE 3D-FLAIR technique of the half brain volume is useful to evaluate the patients with facial neuritis as adjunct to CE 3D-T1WS to improve diagnosis accuracy. Therefore, the authors recommend CE 3D-FLAIR technique of the half brain volume to evaluate other cranial neuropathy.

\section{ACKNOWLEDGEMENT}

This work was supported by the research grant of the Jeju National University Hospital in 2015.

\section{REFERENCES}

1. Hwang JY, Yoon HK, Lee JH, Yoon HM, Jung AY, Cho YA, et al. Cranial nerve disorders in children: MR Imaging Findings. Radiographics 2016;36:1178-94.

2. Kim IS, Shin SH, Kim J, Lee WS, Lee HK. Correlation between MRI and operative findings in Bell's palsy and Ramsay Hunt syndrome. Yonsei Med J 2007;48:963-8.

3. Lim HK, Lee JH, Hyun D, Park JW, Kim JL, Lee HY, et al. MR diagnosis of facial neuritis:diagnositic performance of contrast-enhanced 3D-FLAIR technique compared with contrast enhanced 3D-T1-FFE with fat suppression. AJNR Am J Neuroradiol 2012;33:779-83.

4. Chung MS, Lee JH, Kim DY, Lim YM, Ahn JH, Sung YS, et al. The clinical significance of findings obtained on 3D-FLAIR MR imaging in patients with Ramsay-Hunt syndrome. Laryngoscope 2015;125:950-5.

5. Maeda M, Tsuchida C. 'Ivy sign" on FLAIR images in childhood Moyamoya disease. AJR 1999;20:1836-8.

6. Fukuoka H, Hirai T, Okuda T, Shigematsu Y, Sasao A, Kimura E, et al. Comparison of the added value of contrast-enhanced 3D fluid-attenuated inversion recovery and magnetization-prepared rapid acquisition of gradient echo sequences in relation to conventional postcontrast T1-weightedimages for the evaluation of leptomeningeal diseases at 3T. AJNR Am J Neuroradiol 2010;31:868-73.

7. Bozzao A, Floris R, Fasoli F, Fantozzi LM, Colonnese C, Simonetti G. Cerebrospinal fluid changes after intravenous injection of gadolinium chelate:assessment by FLAIR MR imaging. Eur Radiol 2003;13:592-7.

8. Vrabec JT, Backous DD, Djalilian HR, Gidley PW, Leonetti JP, Marzo SJ, et al. Facial Nerve Disorders Committee. Facial Nerve Grading System 2.0. Otolaryngol Head Neck Surg 2009;140:44550 .

9. Koch BN, Hamilton BE, Hudgins P, Harnsberger HR. Head and neck imaging, $3^{\text {rd }}$ eds, New York Elsevier 2016:1166-9.

10. Naganawa S, Komada T, Fukatsu H, Ishigaki T, Takizawa O. Observation of contrast enhancement in the cochlear fluid space of healthy subjects using a 3D-FLAIR sequence at 3 Tesla. Eur Radiol 2006;16:733-7.

11. Gebarski SS, Telian SA, Niparko JK. Enhancement along the normal facial nerve in the facial canal: MR imaging and anatomic correlation. Radiology 1992;183:391-4.

12. Balkany I, Fradis M, Jafek BW, Rucker NC. Hemangioma of the facial nerve: role of the geniculate capillary plexus. Skull Base Surg 1991;1:59-63.

13. Naganawa S, Koshikawa T, Nakamura T, Kawai H, Fukatsu H, Ishigaki T, et al. Comparison of flow artifacts between 2D-FLAIR and 3D-FLAIR sequences at 3 T. Eur Radiol 2004;14:1901-8.

14. Mathews VP, Caldemeyer KS, Lowe MJ, Greenspan SL, Weber DM, Ulmer JL. Brain:gadolinium-enhanced fast fluid-attenuated inversion-recovery MR imaging. Radiology 1999;211:257-63.

15. Chagla GH, Busse RF, Sydnor R, Rowley HA, Turski PA. Three-dimensional fluid attenuated inversion recovery imaging with isotropic resolution and nonselective adiabatic inversion provides improved three-dimensional visualization and cerebrospinal fluid suppression compared to two-dimensional flair at 3 tesla. Invest Radiol 2008;43:547-51.

16. Mamourian AC, Hoopes PJ, Lewis LD. Visualization of intravenously administered contrast material in the CSF on fluid-attenuated inversion-recovery MR images: an in vitro and animal-model investigation. AJNR Am J Neuroradiol 2000;21:105-11 . 